\title{
Can home-based rehabilitation services facilitate rehabilitation transition from hospital to community?
}

\author{
Iris Lee ${ }^{1}, \mathrm{PhD}, \mathrm{RN}$, Felix Yau $^{2}$, MSc, PT, Sally Yim² ${ }^{2}$ MA, OT, Diana Lee ${ }^{1} \mathrm{PhD}, \mathrm{RN}$, \\ FAAN, JP \\ 1 The Nethersole School of Nursing, The Chinese University of Hong Kong \\ 2 Nethersole Outreaching Rehabilitation Mission \\ Correspondence to: Prof Iris Lee. Email: fk95lee@cuhk.edu.hk
}

\section{To the Editor:}

In Hong Kong, the overload of the health care system and the heavy burden on caregivers urge the revisit of the local community rehabilitation services. Owing to the ageing population, high demand for hospital beds inevitably leads to early discharge of patients. Post-discharge rehabilitation services thus become exceptionally important to older patients. The transition period from hospital to home is critical for rehabilitation, as it affects patient recovery and adjustment to daily activities and quality of life. ${ }^{1}$ In addition, the stress of caregivers should not be overlooked. For instance, in 2017 an old man killed his wife, who had physical deconditioning at home, because of stress from prolonged caregiving. ${ }^{2}$ To prevent such tragedies, rehabilitation services should provide caregiver support such as skill training and referral to social services. However, there are gaps in the existing subsidised rehabilitation services for the older people in Hong Kong.

The Hospital Authority is the major provider for active rehabilitation service. It has recently extended its post-discharge rehabilitation from hospitalbased services to home-based services by adopting the Integrated Care Model and the Medical-Social Collaboration. Priority of home-based services is given to selected patients such as those with high readmission risk for a short period only (eg,
8 weeks). However, older patients usually require a longer active rehabilitation to maximise their functional independence and prevent unwarranted deterioration. The existing active rehabilitation services may not be sufficient, especially for those newly discharged and those unable to attend centre-based rehabilitation services. Moreover, the community-based subsidised services for older people (such as the Enhanced Home and Community Care Service, the Integrated Home Care Service, and the Community Care Service Voucher organised by non-government organisations) are mainly designed for supporting homecare needs with less emphasis on active rehabilitation. Only moderately to severely frail older people receive low-frequency rehabilitation services. Hence, community-dwelling older patients requiring active rehabilitation may not have adequate rehabilitation services. In addition, these long-term care services have a long waitlist owing to the ageing population. Some older patients who cannot continue to receive post-discharge rehabilitation services may have missed the golden rehabilitation period and are thus at risk of irreversible physical deconditioning.

The existing community rehabilitation services cannot fill the service gaps for active rehabilitation. Patient selection and service inflexibility further widen the service gaps of community active rehabilitation. More importantly, community service providers have 
difficulty accessing the patient's medical information owing to organisation separation. They also lack communication channels with hospital service providers and health care service support. These are barriers to follow up patients in the community.

The Home-Based Rehabilitation Service (HBRS) of the Nethersole Outreaching Rehabilitation Mission is a community service with connection to a district hospital to facilitate a seamless transition of rehabilitation service from hospital to home using a double-based (hospital- and communitybased) model. ${ }^{3}$ The HBRS staff belong to a nongovernmental organisation, but the office is located in the hospital. By means of honorary appointments, HBRS staff are eligible to access to the Hospital Authority Clinical Management System and participate in case conferences. Such arrangements enhance collaboration with the hospital staff to facilitate smooth transition of patients for rehabilitation. The HBRS staff can also make referrals for patients to different hospital departments. In the community, the HBRS is embedded in the non-governmental organisation network and participates in health promotion activities provided by social care services of the district. The HBRS staff have close communication with the social service providers to direct the patients and their caregivers to appropriate social care services. This double-based model has enabled an effective hospital-community partnership.

The HBRS fills the service gap by providing home-based rehabilitation services to patients and their caregivers in a timely and flexible manner. With assistance of HBRS staff, patients can continue active rehabilitation immediately after hospital discharge, and the number of rehabilitation sessions is suggested by physiotherapists or occupational therapists of the HBRS with the agreement of the individual patients. The HBRS staff aim to assist patients to attain optimal recovery and to seamlessly transfer to other appropriate services (eg, Enhanced Home and Community Care Service).

TheHBRShasbeenevaluatedbyamatched-control quasi-experimental study with in-depth individual interviews with users and service providers. ${ }^{4,5}$ Elderly patients using HBRS were compared with elderly people recruited from Integrated Home Care Service and community centers in the same district. Both groups were matched by age, gender, chronic disease, frailty level, and the presence of a caregiver. The HBRS group demonstrated significant improvement in all quantitative health outcomes including the mobility, physical functioning, cognitive functioning, quality of life, and hospital service utilisation, and the caregiver strain. In addition, the intervention group had enhanced motivation for rehabilitation participation, and their caregivers had better physical and psychosocial well-being, and their service providers had better job satisfaction.

In conclusion, a double-based rehabilitation service model is recommended. Establishment of a rehabilitation service office in a hospital of the serving district with honorary appointment of staff is essential to promote communication between hospital and community health care professionals. Moreover, therapists should be able to prescribe a course of rehabilitation that best suits the needs of patients. The rehabilitation service should establish close collaboration with the social service providers of the district.

\section{REFERENCES}

1. Stott DJ, Quinn TJ. Principles of rehabilitation of older people. Medicine 2017;45:1-5. Crossref

2. Lo C. Hong Kong man who 'strangled disabled wife feared no one would take care of her if he fell sick'. South China Morning Post, 6 Jun 2017. Available from: http://www.scmp. com/news/hong-kong/law-crime/article/2097079/manstrangled-disabled-wife-hong-kong-flat-end-her. Accessed 30 September 2018.

3. Alice Ho Miu Ling Nethersole Charity Foundation. Nethersole 
Outreaching Rehabilitation Mission. Available from: http:// www.nethersole.org.hk/catalog/info.php?target=service_01a\&la nguage $=$ en $\&$ osCsid=ueqk8hq0nj865iiberk1kqpcq5. Accessed 30 September 2018

4. Lee IF, Yau FN, Yim SS, Lee DT. Evaluating the impact of a homebased rehabilitation service on older people and their caregivers: a matched-control quasi-experimental study. Clin Interv Aging
2018;13:1727-37. Crossref

5. Lee DT, Lee IF. Evaluating the effectiveness of the HomeBased Rehabilitation Service (HBRS) Model of the Nethersole Outreaching Rehavilitation Mission (NORM): a mixed methods study. A final report to the Alice Ho Miu Ling Nethersole Charity Foundation. Hong Kong: The Nethersole School of Nursing, The Chinese University of Hong Kong; 2017. 